\title{
Breeding and life cycle of Neocaridina denticulata sinensis (Kemp, 1918).
}

\begin{abstract}
Neocaridina denticulata sinensis (Kemp, 1918) or also known as red cherry shrimp (RCS) became popular in aquarium industry since its first introduction in 2003 until now. Little is known of this particular species since documented report is very scarce. Thus, this study was conducted to gather some information on its breeding behavior and life cycle until first maturity in laboratory condition. Fertilized eggs were found to be oval in shape with color varies from greenish to yellowish. The egg size was comparatively large, with an average diameter of $1.19 \mathrm{~mm}$. Embryonic development of N. d. sinensis lasted for 15 days at $27^{\circ} \mathrm{C}$. Newly hatched larvae look like a miniature version of the adult with an average total length of $2.3 \mathrm{~mm}$. A female can produced about 21-51 larvae per hatching. Larger females produced more larvae. It takes 60 days for larvae to reach juvenile stage, where male and female were still undifferentiated. Juveniles become adults 15 days later. Females were obvious with the presence of orange colored ovary at the cephalothorax region. Within one to three days, these males and females are ready to spawn. During this study, N. d. sinensis cultured in freshwater were found to be susceptible to clitellate annelid (Holtodrilus sp.). Culture of this species in slightly saline condition between 5-10 ppt was an effective treatment for this annelid.
\end{abstract}

Keyword: Breeding; First maturity; Life cycle; Neocaridina denticulata sinensis. 